Ann. Biol. anim. Bioch. Biophys., I973, 13 (3), 4I9-427.

\title{
MÉTABOLISME DE LA MYOSINE DANS TROIS MUSCLES STRIÉS DE TYPE DIFFÉRENT, CHEZ LE LAPIN
}

\author{
A. SIGNORET, P. VIGNERON et J. MORETTI \\ Laboratoires de Chimie biologique et de Biochimie, \\ Université des Sciences et Techniques du Languedoc, \\ 34060 Montpellier \\ Laboratoire de Zootechnie, \\ École nationale supérieure agronomique, \\ Centre de Recherches de Montpellier, I. N. R. A., \\ 9 , place Viala, \\ 34060 Montpellier
}

\begin{abstract}
RÉSUMÉ
Le métabolisme de ia myosine est étudié dans les muscles cardiaque, soléaire et long dorsal, chez le Lapin. Une injection unique de L-leucine- ${ }^{14} \mathrm{C}$ est pratiquée, suivie d'injections régulières de leucine non marquée. L'incorporation du marqueur dans la myosine varie suivant le muscle considéré et on observe des valeurs décroissantes pour le cœeur, le soléaire, le long dorsal. Cependant les demi-vies déterminées, de l'ordre de 28 jours. ne diffèrent pas de façon significative. Ces résultats sont discutés en tenant compte des diverses méthodes et des valeurs rapportées dans la littérature.
\end{abstract}

La détermination de la vitesse de biosynthèse ou de catabolisme des protéines musculaires pose un problème complexe, car de nombreux facteurs interviennent, comme par exemple l'état physiologique de l'animal, la nature du muscle (rouge, mixte ou blanc), le choix de l'acide aminé marqué, ou enfin la réincorporation de ce marqueur provenant du catabolisme soit des protéines musculaires elles-mêmes, soit des autres protéines de l'organisme. Il n'est donc pas surprenant que les valeurs trouvées pour la demi-vie des protéines myofibrillaires chez la Souris (SImon et coll., I 962), le Rat (DREYFus et coll., I960 ; WATERLow et STEPHEN, I968 ; GarLICK, I969; GAN et JEFFAY, I97I) et le Chien (Yokota, I967) soient très différentes, et que, chez une même espèce animale, le Lapin, les valeurs de la demi-vie de la myosine soient parfois diszordantes :

- VeLICK (1956) : 80 jours pour HMM (heavy-meromyosin). 20 jours pour LMM (light-meromyosin), 
- Mc Manus et Muelifer (Ig66) : 20 jours pour la myosine squelettique (HMM ou L,MM),

- Kimata et MoRkin (I97I) : 7,6 jours pour la myosine de cour, I48 jours pour la myosine de muscle rouge et 165 jours pour celle de muscle blanc.

Nous nous sommes alors proposé d'étudier le métabolisme de la myosine dans différents muscles de Lapin en tenant compte de ces facteurs.

\section{MATÉRIEL ET MÉTHODES}

\section{A. - Matériel animal}

Nous avons choisi d'opérer sur un nombre restreint d'animaux en prêtant une attention particulière à leur sélection. L'expérience est réalisée sur 3 lapins adultes femelles, de souche commune, provenant d'une même portée et pesant $4, \mathrm{I} \pm 0,2 \mathrm{~kg}$ (élevage de la station de Zootechnie de l'E. N. S. A. de Montpellier). Ce poids n'a pratiquement pas varié au cours de l'expérience.

Les animaux, à jeun depuis 24 heures, reçoivent chacun $85 \mu \mathrm{Ci}$ de L-leucine ${ }^{14} \mathrm{C}$ uniformément marquée (C. E. A., I $50 \mathrm{mCi} / \mathrm{mM}$ ) par injection dans la veine marginale de l'oreille. Ils sont ensuite nourris avec un aliment granulé (Usine d'Alimentation rationnelle) et reçoivent en outre 4,6 mM de leucine froide chaque jour, dès le lendemain de l'injeotion ; cette valeur correspond à 25 p. 100 des besoins quotidiens de l'animal en leucine.

A des temps variables après l'administration du marqueur $(3,6 ; 11,6$ et 31,6 jours), les animaux sont anesthésiés (I lapin par stade) par injection intraveineuse de pentobarbital sodique (30 $\mathrm{mg} / \mathrm{kg}$ de poids vif) et reçoivent de la $d$-tubocurarine $(6 \mathrm{mg} / \mathrm{kg}$ de poids vif) qui réduira les fibrillations musculaires. Ils sont ensuite rapidement sacrifiés par exsanguination.

L'animal est aussitôt dépouillé. Les muscles à étudier sont prélevés et placés dans la glace pilée. Ce sont :

- Longissimus dorsi, muscle squelettique mixte; deux parties de $20 \mathrm{~g}$ environ.

- Soleus, muscle squelettique rouge, en totalité.

- Le muscle cardiaque entier.

\section{B. - Préparation de la myosine}

Plusieurs méthodes ont été envisagées ; celle de QuASS et BRTSKeY (I968) a été retenue. La myosine est extraite par la solution Guba Straub (tampon phosphate o, $15 \mathrm{M} ; \mathrm{Krll} 0,3 \mathrm{M} ; \mathrm{pH} 6,5$ ) et purifiée au cours de 3 cycles de précipitation-dissolution, par modification de la force ionique. de la solution protéinique.

La myosine ainsi isolée présente un degré de pureté élevé, déterminé à l'aide des critères suivants : le rapport des absorbances à 280 et $260 \mathrm{~nm}$, la mesure du coefficient d'absorbance. $\mathrm{E}_{280}^{1 \%}$, le phénomène de superprécipitation et l'électrophorèse en gel d'acrylamide.

$s$

\section{C. - Extraction des acides aminés libres et des protéines totales}

A partir de $20 \mathrm{~g}$ de tissu musculaire , on extrait les acides aminés libres par l'acide trichloracétique à ıo p. Ioo, à froid. Ensuite, les résidus de l'extraction sont traités et homogénéisés sous forme de poudres qui renferment les protéines totales de l'échantillon (ARNAI et coll., I97I).

Cette préparation est réalisée uniquement à partir du muscle long dorsal. Des 3 muscles choisis, il est le seul à présenter un poids suffisamment important.

\section{D. - Dosages}

La concentration en acides aminés de l'extrait trichloracétique et la teneur en azote total des poudres sont estimées par le dosage de l'azote selon Kjeldahl.

La concentration én myosine des solutions diluées est déterminée par la réaction du biuret (GoRNALL et coll., 1949). 


\section{E. - Mesures de radioactivité}

Elles sont effectuées avec un compteur à scintillation liquide Packard, Tri-Carb modèle 3320. Deux mélanges scintillants sont utilisés :

a) PPO ( $5 \mathrm{~g})$, diméthyl POPOP $(0,3 \mathrm{~g})$ par litre de toluène ;

b) PPO (4 g), diméthyl POPOP $(0,5 \mathrm{~g})$, naphtalène $(80 \mathrm{~g})$ pour $500 \mathrm{ml}$ de dioxane et $500 \mathrm{ml}$ de xylène.

Pour chaque échantillon, deux prises d'essai au moins sont comptées pendant to et 20 minutes Les rendements, mesurés par la méthode du standard externe, sont de l'ordre de 50 à $80 \mathrm{p}$. Ioo.

Pour les extraits liquides (myosine, acides aminés), l'échantillon correspondant à 5-1o mg de protéine est desséché à l'étuve, solubilisé par $1 \mathrm{ml}$ de Soluène pendant 24 heures à $60^{\circ} \mathrm{C}$. et compté avec le premier mélange scintillant.

Pour les poudres, un poids de Io à $20 \mathrm{mg}$ est dissous dans I $\mathrm{ml}$ d'hydroxyde d'hyamine à $60^{\circ}$ pendant 24 heures. On ajoute deux volumes de dioxane. Un ml du mélange est compté avec ro ml du second mélange scintillant.

\section{F. - Électrophorèse en gel d'acrylamide}

L'électrophorèse est réalisée avec l'appareil Canalco $\mathrm{n}^{\circ}$ 6, en tampon Tris-glycine $\mathrm{pH} 8,3$, suivant la technique de Davis (1964).

Les préparations de protéine sont soumises à l'électrophorèse sur des gels de polyacrylamide à $7,5 \mathrm{p}$. 100 et sans dodécyl sulfate de sodium.

\section{RÉSULTATS}

Nous comparerons d'abord la radioactivité spécifique des protéines totales à celle des acides aminés libres du muscle long dorsal. Ensuite, nous évaluerons la demi-vie de la myosine à partir de la courbe de décroissance de la radioactivité spécifique incorporée en fonction du temps, représentée en coordonnées semi-logarithmiques.

\section{A. - Cas du long dorsal}

Les radioactivités spécifiques des acides aminés libres et des protéines totales du muscle long dorsal sont déterminées sur chacun des animaux sacrifiés. A chaque stade, la mesure de radioactivité est effectuée sur 4 prises d'essai.

La figure I représente la radioactivité spécifique, exprimée en d.p.m./mg de

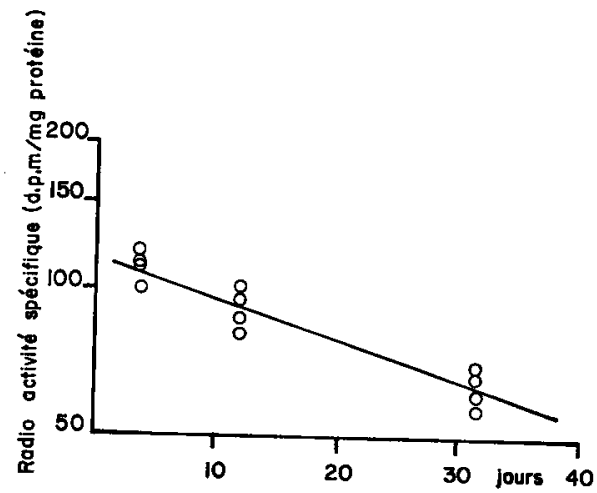

FIG. I. - Evolution de la radioactivité spécifique des protéines totales du long dorsal en fonction du temps (coordonnées semi-logarithmiques)

A chaque stade, les comptages sont réalisés à partir de 4 prises d'essai du môme échantillon 
protéine, des protéines totales préparées à chacun des 3 stades. La droite ainsi tracée permet de calculer, pour les "protéines totales" une demi-vie de $36 \pm 4$ jours.

Dans le tableau I, nous indiquons la radioactivité en d.p.m. des acides aminés libres et des protéines totales du long dorsal rapportée à I mg d'azote ( $\mathrm{I}^{\mathrm{re}}$ et $2^{\circ}$ colonnes) et à I $\mathrm{g}$ de muscle frais ( $3^{\mathrm{e}}$ et $4^{\mathrm{e}}$ colonnes).

\section{TABLEAU I}

Radioactivité spécifique des acides aminés libres et des protéines totales du muscle long dorsal

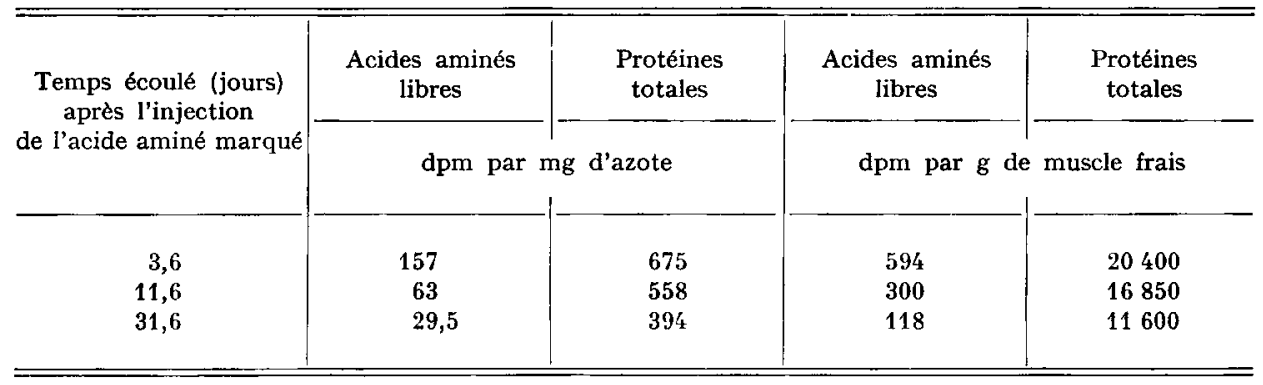

\section{B. - Demi-vie de la myosine}

Pour chaque animal, la myosine est extraite de trois muscles différents : un muscle squelettique mixte, un muscle squelettique rouge et le muscle cardiaque.

La myosine obtenue est généralement pure. Le rapport des absorbances, DO à $278 / \mathrm{DO}$ à $260 \mathrm{~nm}$, est égal à $\mathrm{I}, 7 . \mathrm{A} \mathrm{pH} \mathrm{7,} \mathrm{dans} \mathrm{un} \mathrm{milieu} \mathrm{KCl} 0,5 \mathrm{M}$, Tris- $\mathrm{HCl} 20 \mathrm{mM}$, son coefficient d'absorbance $\mathrm{E}_{278}^{1 \%}=5,7$. En électrophorèse, elle donne une seule bande qui se déplace très peu ; ce résultat est conforme à celui de RAMPToN et coll-(I97I).

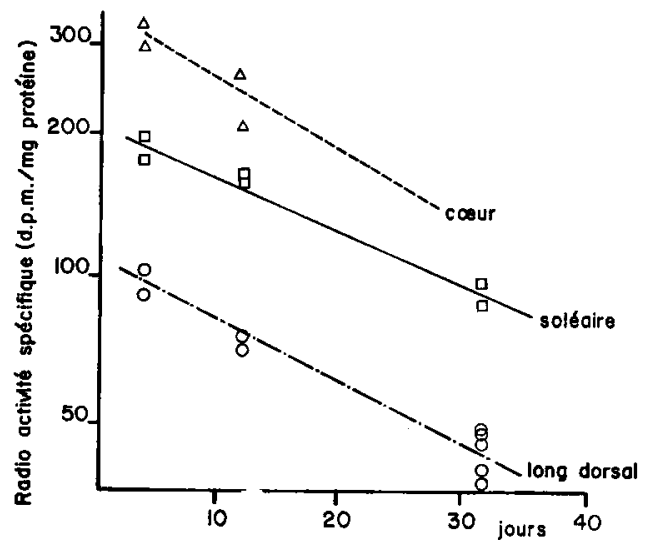

Fig. 2. - Evolution de la radioactivité spécifique de la myosine

en fonction au temps après l'injection de L-leucine ${ }^{14} \mathrm{C}(\mathrm{H})$ (coordonnées semi-logarithmiques)

Par stade et par muscle,

chaque point représente la mesure effectuée à partir de prises d'essai provenant du même échantilloב

Les mesures de radioactivité sont effectuées sur au moins 2 prises d'essai d'un même échantillon. La figure 2 rassemble les résultats obtenus. On voit d'abord que les radioactivités spécifiques (dpm/mg de myosine) ne sont pas identiques. Le cour 
incorpore davantage que le soléaire qui incorpore lui-même plus que le long dorsal. Mais la demi-vie estimée de la myosine diffère peu selon le muscle étudié. Elle est de:

$$
\begin{aligned}
& 30 \pm 2 \text { jours dans le soléaire } \\
& 25 \pm 2 \text { jours dans le long dorsal }
\end{aligned}
$$

Nous ne pouvons pas évaluer de façon précise la demi-vie de la myosine cardiaque, les résultats expérimentaux faisant défaut au stade 31,6 jours.

\section{DISCUSSION}

\section{A. - Comparaison des radioactivités spécifiques des acides aminés libres et des protéines totales du long dorsal}

Selon Hider et coll. (I969, I971), à partir d'expériences réalisées in vitro, la synthèse protéique musculaire se ferait directement à partir du pool des acides aminés circulants ou d'un pool d'acides aminés en rapide équilibre avec ceux-ci. L'administration quotidienne de leucine non marquée a donc certainement facilité l'élimination de la leucine ${ }^{-14} \mathrm{C}$ circulante provenant du catabolisme des protéines initialement marquées et réduit ainsi, dans une certaine mesure, la réutilisation du marqueur.

En effet, d'après le tableau $I$, la radioactivité des acides aminés libres est très faible en comparaison de celle des protéines totales. Par gramme de poids frais, elle représente de 3 à $\mathrm{I}$ p. roo seulement.

On ne peut, malgré tout, éliminer l'hypothèse d'une réutilisation intracellulaire d'acides aminés provenant de la dégradation des protéines (KLEVECZ, I97I). Par infusion constante de lysine ${ }^{14} \mathrm{C}$, GaN et JEFFAY (I967) ont montré, chez le Rat, que ces acides aminés représentent jusqu'à 30 p. Ioo du pool total d'acides aminés intracellulaires. Il nous est apparu difficile d'extrapoler ces résultats à une autre espèce. Il n'a donc pas été fait de correction pour tenir compte de cet aspect du mátabolisme cellulaire.

A cette restriction près, les courbes de décroissance de radioactivité des protéines totales du long dorsal et des diverses myosines, en fonction du temps, nous permettent d'en mesurer le catabolisme.

\section{B. - Incorporation de l'acide aminé marqué}

La leucine est considérée comme non toxique pour l'organisme. En injection intrapéritonéale, une dose quotidienne de $0,76 \mathrm{mM}$ à des rats de $55 \mathrm{~g}$ n'a entraîné aucun ralentissement de croissance (DANIEL et WAISMaN, I968). Da plus, la dégradation de cet acide aminé s'effectue essentiellement dans la masse musculaire. Au cours de notre manipulation, les quantités de leucine non marquée injectées représentent environ $25 \mathrm{p}$. Ioo des besoins quotidiens en cet acide aminé. Nous pənsons ainsi avoir évité une surcharge susceptible d'entraîner une accélération du métabolisme des protéines musculaires signalée par KIMATA et MoRkIN (I97I), MORGAN et coll. (I97I) dans le muscle, par HANking et Roberts (I965) dans le foie. Cependant 
nous ne pouvons éliminer totalement l'hypothèse d'une stimulation dê la synthès? protéique liée aux propriétés insulino-sécrétrices de la leucine.

Les acides aminés alimentaires ne sont pas tous utilisés diractemənt pour la synthèse protéique. Une accumulation importante se fait dans le muscle, suivie d'un recyclage (ARNAI et coll., I97I). Par ailleurs, chez le Lapin, les prises de nourriture sont multiples et réparties sur la totalité du nycthémère (PRUD'HON et coll., I972). Pour ces raisons et de façon à amoindrir les réserves tissulaires en acides aminés libres, les animaux ont été mis à jeun $24 \mathrm{~h}$ avant l'injection du marqueur. De plus, cette injection a été pratiquée à $\mathrm{I} 8 \mathrm{~h}$ afin de favoriser l'incorporation chez cet animal qui présente un maximum d'activité crépusculaire ou nocturne. En effet le niveau de synthèse protéique suit le niveau d'activité de l'animal (GAGLIARDINo et REBoLLÉDO, I972).

I,a synthèse protéique peut présenter des variations quotidiennes liées aux fluctuations cycliques de $24 \mathrm{~h}$ des concentrations plasmatiques en acides aminés (WuRTMAN, I970). Par ailleurs, des protéines à turnover rapide comme celles du foie atteignent plus rapidement une plus grande activité spécifique que les protéines musculaires et progressivement un équilibre isotopique tend à s'établir entre les différents organes. La diminution rapide de la radioactivité spécifique des acides aminés circulants reflète ce phénomène. En vue d'atténuer l'effet de ces variations, et pour mesurer une incorporation maximale dans les protéines, nous avons effectué les premiers prélèvements trois jours après l'injection. A ce moment, les radioactivités spécifiques de la myosine des différents muscles, proportionnelles aux vitesses d'incorporation apparente, sont dans l'ordre décroissant : cour, soléaire, long dorsal.

Ces résultats rejoignent ceux de divers auteurs concernant soit le muscle entier (BIron et coll., I964; GuTmann et Syrovy, I967 ; Yokota, I967), soit la myosine proprement dite (KIMATA et MoRKIN, I97I).

Cette incorporation plus grande dans les protéines de muscle rouge et de cour peut refléter une pénétration plus rapide, par perméabilité ou surface membranaire accrue, de l'acide aminé marqué dans le pool d'acides aminés libres intracellulaires telle qu'elle a déjà été rapportée in vivo (DREYFus, I965; GoldBERG, I967). Ainsi, 1'irrigation sanguine supérieure du muscle rouge (Romanul, Ig65) pourrait en rendre compte, en partie. Ces vitesses d'incorporation différentes pourraient alors être interprétées de diverses façons:

I $^{\circ}$ La vitesse de synthèse de la myosine est identique quel que soit le muscle, mais les échanges plus importants dans le muscle rouge diminuent la part relative des acides aminés provenant de la dégradation des protéines et réutilisés in situ. Ceci serait en accord avec les travaux de Wool et coll. (I958), in vitro, qui montrent une même capacité de synthèse pour des ribosomes provenant de ces deux typas de muscles.

$2^{\circ}$ La vitesse de synthèse protéique est plus rapide dans le muscle rouge indépendamment de toute autre considération. Ce qui semble confirmé par SHOR'T (I969) qui observe in vitro une vitesse d'incorporation plus élevée de méthionine-14C dans les protéines de fibres rouges. De plus, il semble que les diverses activités métaboliques soient plus intenses au niveau du muscle rouge caractérisé, en particulier, par un métabolisme énergétique de caractère essentiellement oxydatif.

La suite de la discussion va nous permettre de choisir entre ces 2 hypothèses. 


\section{C. - Catabolisme de la myosine}

La dégradation de différentes protéines chez un animal en état d'équilibre métabolique, tel que ceux que nous avons choisi d'étudier, doit varier dans le même sens que la synthèse de ces protéines. C'est ce qui ressort en particulier du travail de KIMATA et MoRkin (I97I). Cependant, alors que nous observons des taux d'incorporation très différents dans les trois muscles choisis, nos résultats, bien qu'obtenus à partir d'un nombre réduit d'animaux, ne nous permettent pas d'attibuer une demivie différente aux myosines de muscle cardiaque, rouge ou mixte. Le catabolisme de la myosine semblerait ainsi comparable dans les trois muscles considérés. Par suite, nous pourrions en déduire un anabolisme semblable quel que soit le type de muscle, ce qui permettrait de retenir la première hypothèse formulée.

L'accélération du métabolisme des protéines signalée par KIMATA et MORKIN (I97I) et d'autres auteurs cités pourrait s'interpréter de façon analogue, la réutilisation intracellulaire in situ étant diminuée, dans ce cas, par la surcharge en acides aminés exogènes qui augmente les échanges.

Dans cette optique, on peut d'ailleurs observer que les demi-vies, estimées par l'étude de l'anabolisme avec perfusion continue d'acides aminés marqués, sont généralement plus courtes que celles déterminées à partir de l'étude du catabolisme sans " chase ". Il est vraisemblable aussi, que ces différences sont accentuées par l'utilisation, dans de nombreux cas, d'animaux en croissance. C'est ainsi que MrLLWARD (I970), à la suite d'une seule injection de carbonate- ${ }^{14} \mathrm{C}$ chez le Rat, et étudiant dans le muscle les variations d'activité, spécifique ou totale, de deux acides aminés peu réutilisés (acide aspartique, acide glutamique) trouve des valeurs de demi-vie, pour les protéines musculaires totales, proches de celles obtenues par perfusion : 5 à 7 jours, par WATERLów et STEPHEN (I968) et par GaRLICK (I969). Ces résultats (WATERLow et STEPHEN, I968; GARLICK, I969; MrLLWARD, I970) sont obtenus avec des rats n'ayant pas terminé leur croissance pondérale. Par contre GaN et JEFFAy (I97I), opérant également par perfusion de lysine ${ }^{14} \mathrm{C}$, sur des rats ayant atteint le palier de croissance $\left(250-35^{\circ} \mathrm{g}\right)$, déterminent des demi-vies beaucoup plus importantes : 42 jours.

Chez le Lapin, Velick (I956), Mac Manus et Muelier (I966), Kimata et MORKIN (I97I), ont opéré respectivement sur des animaux pesant 3,7,3,5 et $2,7 \mathrm{~kg}$; nous avons utilisé des lapins de poids voisin $(4 \mathrm{~kg})$. Si les différences d'incorporation que nous notons pour le cœur, un muscle squelettique rouge (soléaire) et un muscle squelettique mixte (long dorsal), vont dans le même sens que celles indiquées par KIMATA et MoRkin (I97I), nos résultats quant à la demi-vie de la myosine sont plus proches de ceux de Mac Manus et MuElLLER, 20 jours (I966). Kimata et Morkin (I97I) obtiennent des demi-vies très différentes pour la myosine cardiaque $(7,5$ jours), et les myosines de muscles squelettiques rouge ou blanc ( $\mathrm{r}_{4} 8$ et $\mathrm{I}_{5}$ jours). Notre travail ne confirme pas cet écart important.

De toute façon, des problèmes essentiels restent posés: D'une part, il serait nécessaire d'étudier de façon plus approfondie le passage d'acides aminés dans le sens tissu-milieu extérieur, au niveau des muscles de divers types. Par ailleurs, la proportion de polyribosomes lourds est faible dans le muscle squelettique d'adulte (SRIVAstava, I969) ; dans le cas du muscle rouge, cette proportion devrait être áccrue si la synthèse de myosine est effectivement plus active dans ce type de muscle. 
Il serait donc intéressant de comparer la distribution des polyribosomes dans le cœur et les muscles squelettiques rouge et blanc. On peut, d'autre part, se demander si la dégradation des protéines se fait bien au hasard. Il semble que 1'on puisse en douter dans certains cas (Sobel, et Bowman, I97I). Le turnover plus rapide de la myosine du myocarde ou du muscle rouge nous le suggère également. Ce turnover plus élevé pourrait simplement refléter une "usure " plus rapide des protéines. Les fibres rougas participent, en effet, à un travail plus fréquent et plus soutenu que les fibres blanches.

Reçu pour publication en mars 1973.

\section{SUMMARY}

\section{MYOSIN METABOLISM IN THREE DIFFERENT TYPES OF STRIATED RABBIT MUSCLE}

Myosin metabolism is studied in the heart and two skeletal muscles, the Soleus (red muscle) and the Longissimus dorsi (mixed muscle), of female rabbits weighing $4 . \mathrm{I} \pm 0.2 \mathrm{~kg}$. Each animal is given an intravenous injection of $85 \mu \mathrm{Ci}$ of L-leucine ${ }^{14} \mathrm{C}$, and then $4.6 \mathrm{mM}$ of non-radioactive L-leucine every day. These rabbits are sacrificed $3.6 ;$ I 1.6 and 3 I.6 days after administration of the label.

Incorporation of the label in the myosin (prepared after QUASS and BRISkEY's metho 1) varies, depending upon the muscle. Decreasing values are found for the heart, Soleus and Longissimus dorsi.

Using curves expressing the decrease of specific myosin radioactivity, the demi-life of this protein is estimated at 28 days without noting any significant difference between the three muscles.

The results are discussed, taking into consideration various methods and values reported in the literature.

\section{RÉFÉRENCES BIBLIOGRAPHIQUES}

Arnal M., Fauconneau G., Pech R., I971. Étude de la synthèse protéique in vivo : mesure de l'activité anabolisante de divers tissus du rat en croissance à l'aide de la L-lysine -14 C (U). Ann. Biol. anim. Bioch. Biophys., 11, 245-264.

Biron P., Drexfus J. - C., Schapira F., 1964. Différences métaboliques entre les muscles rouges et blancs chez le Lapin. C. R. Soc, Biol., 158, I84I-I843.

Daniel R. G., Waisman H. A., 1968 . The effects of excess amino-acids on the growth of the young rat. Growth, 32, 255-265.

Davis B. J., I964. Disc electrophoresis. II. Method and application to human serum proteins. Ann. N. Y. Acad. Sci., 121, 404-427.

Dreyfus J. C., KRUH J., Schapira G., I960. Metabolism of myosin and life time of myofibrils. Biochem. J., '75, 574-578.

DREYFus J. C., I965. La perméabilité des muscles rouges et blanes aux acides aminés. Muscles normaux et atrophiés par section nerveuse. C. R. Soc. Biol., 159, I317-r320.

Gagliardino J. J., Rebolledo O. R., 1972. Hormonal control of protein synthesis in muscle : an approach through the study of its circadian rythm. Horm. Metabol. Res., 4, 278-279.

GAN J. C., JefFAY H., 1967 . Origins and metabolism of the intracellular amino acid pools in rat liver and muscle. Biochim. Biophys. Acta, 148, 448-459.

GAN J. C., JEFFAY H., I97I. The kinetics of transfer of plasma amino acids to tissues, and the turnover rates of liver and muscle proteins. Biochim. Biophys. Acta, 252, I25-135.

GARLICK P. J., I969. Turnover rate of muscle protein measured by constant intravenous infusion of ${ }^{14}$ C-glycine. Nature, Lond., 228, 61-62.

Goldberg A. L., I967. Protein synthesis in tonic and phasic skeletal muscles. Nature, Lond., 216, 1219-1220. 
Gornall A. G., Bardawill C. S., David M. M., I949. Determination of serum proteins by means of the biuret reaction. J. Biol. Chem., 177, 751 .

Gurmann E., Syrovy I., r967. Metabolic differentiation of the anterior and posterior Latissimus dorsi of the chicken during development. Physiologia Bohemoslov., 16, 232-243.

Hankin B. M., RoBerTs S., r965. Stimulation of protein synthesis in vitro by elevated levels of amino acids. Biochim. Biophys. Acta, 104, 427-438.

Hider R. C., Fern E. B., London D. R., 1969. Relationships between intracellular amino acids and protein synthesis in the Extensor Digitorum longus muscle of rats. Biochem. J., 114, I7I-I78.

Hider R. C., Fern E. B., London D. R., 197r. Identification in skeletal muscle of a distinct extracellular pool of amino acids and its role in protein synthesis. Biochem. J., 121, 817-827.

Kimata S. MoRKin E., I97I. Comparison of myosin synthesis in heart and red and white skeletal muscles. Am. J. Physiol., 221, I706-1713.

KLEVECZ R. R., I97I. Rapid protein catabolism in mammalian cells is obscured by reutilization of amino acids. Biochem. Biophys. Res. Commun., 43, 76-8I.

Mc Manus I. R., Muelier H., 1966. The metabolism of myosin and the meromyosins from rabbit skeletal muscle. J. Biol. Chem., 241, 5967-5973.

Millward D. J., I97o. Protein turnover in skeletal muscle. I. The measurement of rates of synthesis and catabolism of skeletal muscle protein using ${ }^{14} \mathrm{C}-\mathrm{Na}_{2} \mathrm{CO}_{3}$ to label protein. Clin. Sci., 39, 577-59o.

Morgan H. E., Earl D. C. N., Broadus A., Wolpert E. B., Giger K. E., Jefferson L. S., I97I. Regulation of protein synthesis in heart muscle. I. Effect of amino acid levels on protein synthesis. J. Biol. Chem., 246, 2152-2162.

Prud'hon M., Carles Y., Goussopoulos J., Koehl P. F., 1972. Enregistrement graphique de consommations d'aliment solide et liquide du lapin domestique nourri ad libitum. Ann. Zootech., 21, $45 \mathrm{I}-460$.

Quass D. W. I., Briskey E. J., I968. A study of certain properties of myosin from skeletal muscle. J. Fd. Sci., 88, I80-187.

Rampton J. H., Pearson A. M., Walker J. E., Kapsalis J. G., I971. Disc electrophoresis of Weber-Edsall extract and actomyosin from skeletal muscle. J. Agric. Fd. Chem., 19, 238-240.

Romanul F. C. A., I965. Capillary supply and metabolism of muscle fibers. Archs. Neurol., Chicago, 12, 497-509.

Short F. A., 1969. Protein synthesis by red and white muscle in vitro : effect of insulin and animal age. Am. J. Physiol., 217, 307-309.

Simon E. J., Gross C. S., Lessel I. M., rg62. Turnover of muscle and liver proteins in mice with hereditary muscular dystrophy. Archs. Biochem. Biophys., 96, 4I-46.

Sobel H., Bowman R., 1971. Is protein catabolism non random? Experientia, 27, I163.

Srivastava U., r969. Polyribosome concentration of mouse skeletal muscle as a function of age. Archs. Biochem. Biophys., 130, I29-139.

Velick S. E., r956. The metabolism of myosin, the meromyosins, actin and tropomyosin in the rabbit. Biochim. Biophys. Acta, 20, 228-236.

WAterlow J. C., Stephen J. M., r968. The effect of low protein diets on the turnover rates of serum, liver and muscle proteins in the rat, measured by continuous infusion of $\mathrm{L}^{14} \mathrm{C}$-lysine. Clin. Sci., 35, 287305.

Wool I. G., Stirewalt W.S., Kurihara K., Low R. B., Baily P., Oyer D., I968. Mode of action of insulin in the regulation of protein biosynthesis in muscle. Recent Progr. Horm. Res., 24, I39-208.

Wurtman R. J., I97o. In Munro H. N., Mammalian protein metabolism, vol. 4, 445-477. Academic Press, New York.

ҮокотА Y., I967. Studies on the turnover of myosardial actomyosin in dozs with experimental aortic insufficiency and the effect of vitamin $\mathrm{B}_{12}$ on actomyosin matabolism. Jap. Circul. J., 31, I843-I848. 